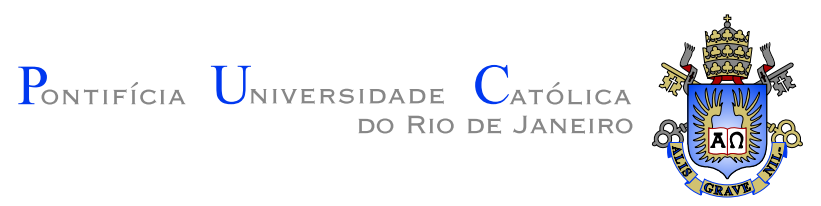

Valéria Quadros dos Reis

\title{
Um estudo sobre reserva de recursos computacionais no nível do usuário
}

Tese de Doutorado

Tese apresentada ao Programa de Pós-graduação em Informática do Departamento de Informática da PUC-Rio como requisito parcial para obtenção do título de Doutor em Informática

Orientador: Prof. Renato Fontoura de Gusmão Cerqueira 


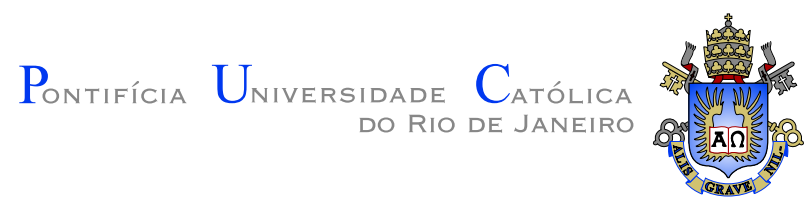

Valéria Quadros dos Reis

\section{Um estudo sobre reserva de recursos computacionais no nível do usuário}

Tese apresentada ao Programa de Pós-graduação em Informática do Departamento de Informática do Centro Técnico Científico da PUC-Rio como requisito parcial para obtenção do título de Doutor em Informática. Aprovada pela Comissão Examinadora abaixo assinada.

Prof. Renato Fontoura de Gusmão Cerqueira
Orientador
Departamento de Informática - PUC-Rio

Profa. Noemi de la Rocque Rodriguez

Departamento de Informática - PUC-Rio

Prof. Sérgio Colcher

Departamento de Informática - PUC-Rio

Prof. Eugene Francis Vinod Rebello

Departamento de Ciência da Computação - UFF

Prof. César Augusto Fonticielha De Rose Faculdade de Informática - PUC-RS

Prof. Antônio Tadeu Azevedo Gomes Coordenação de Sistemas e Redes — LNCC

Prof. José Eugenio Leal

Coordenador Setorial do Centro Técnico Científico - PUC-Rio

Rio de Janeiro, 17 de maio de 2010 
Todos os direitos reservados. É proibida a reprodução total ou parcial do trabalho sem autorização da universidade, do autor e do orientador.

\section{Valéria Quadros dos Reis}

Mestre em Ciência da Computação pela Universidade de São Paulo. Bacharel em Ciência da Computação pela Universidade Federal de Mato Grosso do Sul.

Ficha Catalográfica

Reis, Valéria

Um estudo sobre reserva de recursos computacionais no nível do usuário / Valéria Quadros dos Reis; orientador: Renato Fontoura de Gusmão Cerqueira. - Rio de Janeiro : PUC-Rio, Departamento de Informática, 2010.

v., 100 f: il. ; $29,7 \mathrm{~cm}$

1. Tese (doutorado) - Pontifícia Universidade Católica do Rio de Janeiro, Departamento de Informática.

Inclui referências bibliográficas.

1. Informática - Tese. 2. gerenciamento de recursos. 3. escalonamento de processos. 4. reserva de recursos. 5. provisão de QoS. 6. isolamento de desempenho. 7. nível do usuário. I. Cerqueira, Renato. II. Pontifícia Universidade Católica do Rio de Janeiro. Departamento de Informática. III. Título. 
Aos meus pais, Antônio e Rosângela, com muito amor, carinho e gratidão. 


\section{Agradecimentos}

Agradeço aos meus pais e à minha irmã por terem compartilhado o sonho do doutorado junto comigo. O apoio incondicional da minha família fez com que esse sonho, antes tão distante, pudesse ser alcançado.

Agradeço ao meu marido Eraldo Luís pelo companheirismo que demonstrou nesses anos, sabendo me apoiar, entendendo as minhas variações de humor e lidando com a minha ausência nos momentos em que precisei me dedicar integralmente ao trabalho.

Ao meu orientador, Prof. Renato Cerqueira, quero agradecer pela oportunidade que me deu de cursar o doutorado, pela paciência em me orientar e por entender as minhas limitações acadêmicas. Obrigada por ter acreditado em mim e obrigada pelas palavras de incentivo nos momentos em que eu mesma parecia fraquejar.

Aos meus avós, tios, sogros e primos que torciam e rezavam por mim, obrigada. À Marilda que me acolheu quando cheguei ao Rio, serei sempre grata. Também quero agradecer aos grandes amigos que fiz na PUC, amigos que compartilharam teto comigo, que animavam a hora do almoço, que escutavam e entendiam os meus problemas. Amigos que, muitas vezes, comemoramos juntos.

Quero agradecer também ao Carlos Cassino pela oportunidade de participar do seu grupo de trabalho no Tecgraf e por sempre ter me proporcionado recursos para que eu pudesse realizar um trabalho melhor. Agradeço ainda aos colegas que fiz nesse mesmo laboratório e com os quais aprendi muito.

Por fim, quero agradecer à Capes e ao CNPq por terem financiado esta tese. 


\section{Resumo}

Reis, Valéria; Cerqueira, Renato. Um estudo sobre reserva de recursos computacionais no nível do usuário. Rio de Janeiro, 2010. 100p. Tese de Doutorado - Departamento de Informática, Pontifícia Universidade Católica do Rio de Janeiro.

A forma como a Computação é realizada está mudando devido à grande capacidade de processamento, armazenamento e comunicação que equipamentos computacionais apresentam atualmente. Cenários de compartilhamento de recursos, onde um único servidor físico é compartilhado entre diferentes aplicações, muitas vezes hospedadas em diversos domínios virtuais, têm se tornado comuns, mas demandam esforços para que o isolamento de desempenho de cada aplicação seja garantido como se ela fosse a única a ser localmente executada. Baseado nesse fato, o objetivo deste trabalho consiste em investigar técnicas para a provisão de reservas de recursos, garantindo, consequentemente, qualidade de serviço (QoS) e isolamento de desempenho às aplicações. A fim de atender ambientes onde o uso de extensões de sistemas operacionais ou o uso de virtualização são indevidos ou inapropriados, a investigação contempla o estudo da viabilidade e efetividade de reservas realizadas no nível do usuário, ou seja, sem que instrumentações no núcleo do sistema operacional sejam necessárias. Para isso, implementamos uma ferramenta capaz de limitar e garantir o uso dos recursos de processamento e disco, utilizando somente primitivas providas pelo Sistema Operacional Linux. Entre outras funcionalidades, essa ferramenta apresenta facilidades para a extensão das políticas de escalonamento, o que atribui a ela flexibilidade na forma como os recursos são compartilhados entre os processos. Com a análise de uso da ferramenta, foi possível identificar as vantagens e limitações das técnicas de gerenciamento utilizadas. Como um estudo de caso da ferramenta implementada, parametrizamos reservas para uma aplicação de três camadas com metas de desempenho e verificamos que, mesmo para aplicações complexas, métodos simples tais como a regressão linear são capazes de predizer o uso de recursos com uma baixa margem de erro.

\section{Palavras-chave}

gerenciamento de recursos. escalonamento de processos. reserva de recursos. provisão de QoS. isolamento de desempenho. nível do usuário. 


\section{Abstract}

Reis, Valéria; Cerqueira, Renato (Advisor). A study on computacional resource reservation at user level. Rio de Janeiro, 2010. 100p. DsC Thesis - Departamento de Informática, Pontifícia Universidade Católica do Rio de Janeiro.

The way computing is done today is changing as a result of the everincreasing processing, storage, and communication capacities of modern computer hardware. Resource sharing scenarios, in which a physical server is shared for different applications, are becoming much more common. These scenarios require special attention to guarantee that the performance isolation of each application is carried out exactly as if it were being locally executed. Based on this situation, the present work aims at investigating techniques for providing resource reservations and thus guaranteeing quality of service (QoS) and performance isolation for applications. Considering environments in which the use of operating system extensions or the use of virtualization are unreasonable or inappropriate, this work investigates the viability and effectiveness of reservations done at user level, that is, reservations guaranteed with no operating system kernel instrumentation. For this purpose, we have implemented a tool that limits and ensures the proper usage of processing and disk bandwidth resources through the exclusive use of Linux Operating System primitives, which, among other functions, permits easy scheduling policy extensions. This feature enables flexibility in how resources are shared among distinct processes. Through tool usage analysis, we have identified the advantages and limitations of the techniques used. For a case study, aiming to achieve some specific performance goals, we have established parameter reservations for a three-tier application. We were able to verify that, even for complex applications, simple methodologies like linear regressions are capable of predicting resource usage with a low margin of error.

\section{Keywords}

resource management. resource reservation. QoS provisioning. performance isolation. user level. 


\section{Sumário}

1 Introdução 10

1.1 Objetivos, Abordagem e Contribuições 12

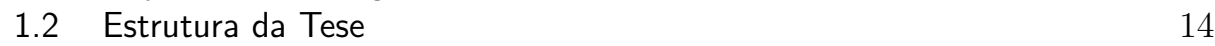

2 Trabalhos Relacionados $\quad 16$

2.1 Provisão de QoS 16

2.2 Considerações Finais 21

3 Uma Proposta para Reserva de Recursos no Nível do Usuário 24

3.1 Arquiteturas de Gerenciamento e a Reserva de Recursos 24

3.2 O Conjunto de Ferramentas ReservationSuite 26

3.2.1 Reserva de Processamento 33

3.2.2 Reserva de Largura de Banda de Disco 36

3.2.3 Reserva de Largura de Banda de Rede 41

3.2.4 Implementação de Novas Políticas 42

3.2.5 Combinando Múltiplas Políticas $\quad 45$

3.3 Considerações Finais 46

4 Experimentos e Avaliação de Desempenho $\quad 50$

4.1 Infraestrutura de Testes $\quad 50$

4.2 Testes de Eficácia da Reserva de Processamento 51

4.3 Escalabilidade do Servidor 52

4.4 Testes de Eficácia da Reserva de Largura de Banda de Disco 54

4.5 Testes de Eficácia da Reserva de Largura de Banda de Rede 59

4.6 Sobrecarga da Interposição de Funções 60

4.7 ReservationSuite e Máquinas Virtuais 62

4.8 Um Estudo de Caso sobre Decomposição de QoS 64

4.8.1 Aplicação Alvo $\quad 67$

4.8.2 Método Adotado 68

4.8.3 Experimentos 71

4.9 Uma Nova Avaliação da Eficácia das Reservas de E/S

4.10 Considerações Finais $\quad 80$

5 Conclusão $\quad 84$

$\begin{array}{lll}5.1 & \text { Trabalhos Futuros } & 87\end{array}$

6 Referências Bibliográficas $\quad 90$ 


\section{Lista de figuras}

3.1 Arquitetura de gerenciamento de recursos. 25

3.2 Arquitetura do ReservationSuite. 30

3.3 Diagrama de caminhos entre os estados de um processo. 34

3.4 Exemplo de sobrescrita da operação de escrita em disco write. $\quad 39$

3.5 Etapas de uma interceptação de chamada ao sistema. 39

3.6 Uma hierarquia de políticas. 47

4.1 Consumo de CPU de aplicações com e sem reserva de processamento. 52

4.2 Uso de CPU do servidor ReservationSuite com um número variável de clientes. 53

4.3 Taxa de escrita de aplicações com e sem reserva de largura de banda de disco.

4.4 Taxa de leitura de aplicações com e sem reserva de largura de banda de disco.

4.5 Taxa de escrita de aplicações com reserva de largura de banda de disco.

4.6 Taxa de envio de dados de aplicação com reserva de largura de banda de rede.

4.7 Consumo de CPU de aplicações com e sem reserva de largura de banda de disco.

4.8 Tempo de execução de aplicações sem limite de uso de disco, com limite imposto por interposição e com limite imposto por interceptação.

4.9 Consumo de CPU de aplicações sem limite de uso de disco, com limite imposto por interposição e com limite imposto por interceptação.

4.10 Hierarquia de reserva de processamento em máquinas virtuais. $\quad 64$

4.11 Representação do método de parametrização. $\quad 69$

4.12 Detalhes do funcionamento de um regressor. $\quad 70$

4.13 Intervalo de confiança para predições. $\quad 70$

4.14 Infraestrutura utilizada nos experimentos de decomposição de QoS. 71

4.15 Consumo de CPU do Tomcat quando essa aplicação não tem o processamento limitado.

4.16 Consumo de CPU do Tomcat quando essa aplicação é limitada a $8 \%$ do processamento total da máquina.

4.17 Consumo de processamento do Tomcat quando os recursos são limitados de acordo com os valores médios da tabela 4.3.

4.18 Médias de WIPS obtidas a partir das regressões baseadas em valores médios e em percentis.

4.19 Vazão obtida pelo TPC-W ao ter o Tomcat e o MySQL executados concorrentemente com outra carga de trabalho. 